\title{
Geometric Ideas for Cryptographic Equation Solving in Even Characteristic
}

\author{
Sean Murphy and Maura B. Paterson ${ }^{\star}$ \\ Information Security Group, Dept. of Mathematics, \\ Royal Holloway, University of London, \\ Egham, Surrey TW20 0EX, U.K.
}

\begin{abstract}
The GeometricXL algorithm is a geometrically invariant version of the XL algorithm that uses polynomials of a much smaller degree than either a standard Groebner basis algorithm or an XL algorithm for certain multivariate equation systems. However, the GeometricXL algorithm as originally described is not well-suited to fields of even characteristic. This paper discusses adaptations of the GeometricXL algorithm to even characteristic, in which the solution to a multivariate system is found by finding a matrix of low rank in the linear span of a collection of matrices. These adaptations of the GeometricXL algorithm, termed the EGHAM process, also use polynomials of a much smaller degree than a Groebner basis or an XL algorithm for certain equation systems. Furthermore, the paper gives a criterion which generally makes a Groebner basis or standard XL algorithm more efficient in many cryptographic situations.
\end{abstract}

Keywords: XL Algorithm, GeometricXL Algorithm, EGHAM process.

\section{Introduction}

The solution of a system of multivariate equations over a field is a problem that has recently attracted much attention in cryptology. The classical method for analysing such an equation system is to calculate its Gröbner basis by using Buchberger's algorithm or a related method [26|7]. Furthermore, other techniques have been proposed for solving a multivariate equation system in a cryptographic context, such as the XL algorithm [5]. However, a Gröbner basis algorithm with the lexicographic ordering and an XL algorithm are closely related [1].

The geometric properties of the XL algorithm are discussed in [12]. In particular, the XL algorithm is not a geometrically invariant algorithm, that is a simple change of co-ordinate system can vastly increase or decrease the running time of the XL algorithm. The GeometricXL algorithm, proposed in [12, is a geometrically invariant algorithm which can solve certain multivariate equation systems using polynomials of a much smaller degree than a Gröbner basis algorithm or

\footnotetext{
^ M.B. Paterson was supported by EPSRC grants GR/S42637 and EP/D053285.
} 
an XL algorithm. However, the GeometricXL algorithm as described in [12] cannot easily be used in a field of even characteristic, which is of course a situation of great cryptographic importance. The main contribution of this paper is to give an adaptation of the GeometricXL algorithm that is specifically tailored for use in a field of even characteristic. This adaptation of the GeometricXL algorithm, like the original GeometricXL algorithm, is one that attempts to find a linear combination of a collection of matrices that has low rank, a problem sometimes termed MinRank. We note that some related issues concerning the MinRank problem in cryptology are considered in [8]. We term the adaptation of the GeometricXL algorithm given in this paper the EGHAM process, and we note that the EGHAM process can solve certain multivariate equation systems in even characteristic using polynomials of a much smaller degree than a Gröbner basis algorithm or an XL algorithm. Furthermore, a criterion (the $\mathcal{L S}$-criterion) used by the EGHAM process generally greatly reduces the number of equations under consideration. This reduction criterion can also be applied directly to a standard Gröbner basis or XL algorithm in many cryptographic situations, so directly making these algorithms far more efficient.

\section{The XL Algorithm}

We consider the polynomial ring $\mathbb{F}\left[x_{0}, \ldots, x_{n}\right]$ of polynomials in $n+1$ variables over a field $\mathbb{F}$. An XL algorithm transforms a homogeneous (without loss of generality) equation system into a homogeneous equation system $f_{1}=\ldots=f_{m}=0$ of degree $D$ by multiplying the original polynomials by selected monomials $[5]$. We generally suppose that this equation system has a unique (projective) solution, a common situation in cryptology, though most of our comments are more generally applicable. The aim of an XL algorithm is to solve this new system by linearisation [5], that is by regarding each monomial of degree $D$ as an independent variable and then applying basic linear algebra. The GeometricXL algorithm [12] exploits the geometrical properties of the new equation system to give a solution method that is most applicable when the field characteristic is either zero or exceeds $D$. We discuss the GeometricXL algorithm in this case in this section and give an alternative description of the GeometricXL algorithm.

\subsection{The GeometricXL Algorithm}

The critical step of the XL algorithm [12] is an attempt to solve a system of homogeneous equations $f_{1}=\ldots=f_{m}=0$ of degree $D$ in a field of characteristic $p$ by finding a bivariate polynomial in $\left\langle f_{1}, \ldots, f_{m}\right\rangle$. However, the set of bivariate polynomials is not invariant under collineation (change of co-ordinates), so the $\mathrm{XL}$ algorithm is not geometrically invariant. The GeometricXL algorithm is a geometric invariant generalisation of the XL algorithm. The GeometricXL algorithm focusses on rank-2 product polynomials (Definition 11), an invariant generalisation of the bivariate polynomial, and the GeometricXL algorithm is motivated by Lemma 11 the geometric invariance result of [12]. 
Definition 1. A rank-2 product polynomial is a homogeneous polynomial of degree $D$ in the polynomial ring $\mathbb{F}\left[x_{0}, \ldots, x_{n}\right]$ of the form

$$
\prod_{i=1}^{D}\left(\theta_{i} L-\theta_{i}^{\prime} L^{\prime}\right)
$$

where $L$ and $L^{\prime}$ are homogeneous linear polynomials and $\theta_{i}, \theta_{i}^{\prime}$ are constants in some extension field $\overline{\mathbb{F}}$ of $\mathbb{F}$. We let $\mathcal{R}_{\mathbb{F}, n}^{D}$ denote the set of all such rank-2 product polynomials of degree $D$ in $\mathbb{F}\left[x_{0}, \ldots, x_{n}\right]$.

Lemma 1. The set $\mathcal{R}_{\mathbb{F}, n}^{D}$ of all rank-2 product polynomials of degree $D$ in the polynomial ring $\mathbb{F}\left[x_{0}, \ldots, x_{n}\right]$ is invariant under collineation.

The GeometricXL algorithm requires us to find a linear combination $g$ of $f_{1}, \ldots$, $f_{m}$ such that $g=\sum_{l=1}^{m} \lambda_{l} f_{l}$ is a rank-2 product polynomial, that is $g \in \mathcal{R}_{\mathbb{F}, n}^{D}$. If such a rank-2 product polynomial can be found, then we know that any solution to the original system $f_{1}=\ldots=f_{m}=0$ satisfies $\theta_{i} L-\theta_{i}^{\prime} L^{\prime}=0$ for some value of $i$. This gives us linear expressions in $x_{0}, x_{1}, \ldots, x_{n}$, which provides information about the solution and potentially allows us to eliminate one variable from the equation system, giving us a smaller equation system and so on.

For any homogeneous polynomial $h$ of degree $d$ and any monomial $\mathbf{x}=$ $x_{0}^{e_{0}} \ldots x_{n}^{e_{n}}$ of degree $k \leq d$ (so $e_{0}+\ldots+e_{n}=k$ ), we denote the $k^{t h}$ order partial derivative of $h$ with respect to $\mathbf{x}$ by $\mathbf{D}_{\mathbf{x}}^{k} h$, so $\mathbf{D}_{\mathbf{x}}^{k} h=\frac{\partial^{k} h}{\partial \mathbf{x}}$. We can now represent all the possible $k^{\text {th }}$ order partial derivatives of $h$ as a matrix in which each row is the polynomial $\mathbf{D}_{\mathbf{x}}^{k} h$ of degree $d-k$ represented as a vector of its coefficients. There are $\left(\begin{array}{c}n+k \\ k\end{array}\right)$ monomials of degree $k$ in $n+1$ variables, so we can define an $\left(\begin{array}{c}n+k \\ k\end{array}\right) \times\left(\begin{array}{c}n+d-k \\ d-k\end{array}\right)$ partial derivatives matrix

$$
C_{h}^{(k)}=\left(\mathbf{D}_{\mathbf{x}}^{k} h\right) .
$$

The GeometricXL algorithm works as a rank-2 product polynomial can be identified using Lemma 2 (given by results of [12]) from its partial derivatives of order $D-1$ in fields of certain characteristics.

Lemma 2. Suppose $g$ is a homogeneous polynomial of degree $D$ in $\mathbb{F}\left[x_{0}, \ldots, x_{n}\right]$, where the field $\mathbb{F}$ has characteristic zero or characteristic exceeding $D$. The polynomial $g$ is a rank-2 product polynomial if and only if the partial derivatives matrix $C_{g}^{(D-1)}$ satisfies

$$
C_{g}^{(D-1)}=\left(\mathbf{D}_{\mathbf{x}}^{D-1} g\right)=\left(a_{\mathbf{x}} L+a_{\mathbf{x}}^{\prime} L^{\prime}\right)
$$

for some homogeneous linear polynomials $L$ and $L^{\prime}$ and constants $a_{\mathbf{x}}$ and $a_{\mathbf{x}}^{\prime}$. An equivalent condition is that for a field $\mathbb{F}$ with charateristic zero or characteristic exceeding $D$, then $g \in \mathcal{R}_{\mathbb{F}, n}^{D}$ if and only if $C_{g}^{(D-1)}$ has rank at most 2. Moreover, if $g \in \mathcal{R}_{\mathbb{F}, n}^{D}$ then $C_{g}^{(D-1)}$ has rank at most 2 in a field $\mathbb{F}$ of any characteristic. 
The GeometricXL algorithm attempts to find some linear combination of $f_{1}, \ldots$, $f_{m}$ such that $g=\sum_{l=1}^{m} \lambda_{l} f_{l} \in \mathcal{R}_{\mathbb{F}, n}^{D}$. The same linear combination of partial derivatives matrices of $f_{1}, \ldots, f_{m}$ satisfies

$$
C_{g}^{(D-1)}=\left(\mathbf{D}_{\mathbf{x}}^{D-1} g\right)=\sum_{l=1}^{m} \lambda_{l}\left(\mathbf{D}_{\mathbf{x}}^{D-1} f_{l}\right)=\sum_{l=1}^{m} \lambda_{l} C_{f_{l}}^{(D-1)} .
$$

This matrix $C_{g}^{(D-1)}$ has rank 2 by Lemma 2 , so all of the 3 -minors $(3 \times 3$ subdeterminants) of $C_{g}^{(D-1)}=\sum_{l=1}^{m} \lambda_{l} C_{f_{l}}^{(D-1)}$ vanish. This gives a system of cubic equations in $\lambda_{1}, \ldots, \lambda_{m}$. For some equation systems and choices of $m$ and $n$, this cubic system is easily soluble, for example by linearisation. This process is in essence the GeometricXL algorithm, and it uses polynomials of a much smaller degree for certain equation systems than either a Gröbner basis or XL algorithm [12].

It is a consequence of Lemma 2 that the performance of the GeometricXL algorithm as originally described in [12] depends greatly on the field characteristic. When the characteristic $p$ of the field $\mathbb{F}$ satisfies $p=0$ or $p>D$, then identifying a partial derivatives matrix of rank at most 2 gives a rank-2 product polynomial (Lemma 2) and so gives information about the solution to the original system. However, when the characteristic $p$ satisfies $0<p \leq D$, then a partial derivatives matrix of rank at most 2 may or may not give a rank- 2 product polynomial. In particular, the GeometricXL algorithm of [12] is not well-suited for fields of even characteristic.

\subsection{An Alternative Description of the GeometricXL Algorithm}

The GeometricXL algorithm as described in 12 requires the use of $(D-1)^{t h}$ order partial derivatives matrices in order to solve a homogeneous multivariate polynomial system of degree $D$. However, we now give an equivalent description of the GeometricXL algorithm in terms of first order partial derivatives matrices when the field characteristic is either zero or it exceeds the degree $D$.

Definition 2. Let $h$ be a homogeneous polynomial of degree $D$ in the polynomial ring $\mathbb{F}\left[x_{0}, \ldots, x_{n}\right]$, where the field has characteristic $p$ and either $p>D$ or $p=0$. Furthermore let $\mathbf{x}=x_{0}^{e_{0}} \ldots x_{n}^{e_{n}}$ denote a monomial of degree $k$ $(0 \leq k \leq D)$, so $e_{0}+\ldots+e_{n}=k$. The $k^{t h}$-order catalecticant matrix 11 of $h$ is

$$
\widetilde{C}_{h}^{(k)}=\left(\frac{1}{e_{0} ! \ldots e_{n} !} \cdot \frac{\partial^{k} h}{\partial x_{0}^{e_{0}} \ldots \partial x_{n}^{e_{n}}}\right)=\left(\frac{1}{e_{0} ! \ldots e_{n} !} \mathbf{D}_{\mathbf{x}} h\right) .
$$

Lemma 3. The catalecticant matrix $\widetilde{C}_{h}^{(k)}$ satisfies $\left(\widetilde{C}_{g}^{(k)}\right)^{T}=\widetilde{C}_{g}^{(d-k)}[11$, and the catalecticant and partial derivatives matrices of order $k, \widetilde{C}_{h}^{(k)}$ and $C_{h}^{(k)}$, share the same row space and have the same rank, with in particular $\widetilde{C}_{h}^{(1)}=C_{h}^{(1)}$.

The GeometricXL algorithm tries to find a rank-2 product polynomial $g$. Lemma 3 shows that the column space of first order partial derivatives 
matrix $C_{g}^{(1)}$ is identical to the row space of the $(D-1)^{t h}$ order partial derivatives matrix $C_{g}^{(D-1)}$, so giving Lemma 4 .

Lemma 4. If the characteristic of $\mathbb{F}$ is either zero or exceeds $D$, then a multivariate polynomial $g$ over $\mathbb{F}$ of degree $D$ is a rank-2 product polynomial, that is $g \in \mathcal{R}_{\mathbb{F}, n}^{D}$, if and only if its first partial derivatives matrix $C_{g}^{(1)}$ has rank 2.

Lemma 4 means that the GeometricXL algorithm could be carried out by using the cubic system derived from the first order partial derivatives matrix rather than the $(D-1)^{t h}$ order partial derivatives matrix. Furthermore, a basis for the column space of $C_{g}^{(1)}$ gives a pair of homogeneous linear polynomials $L$ and $L^{\prime}$ of use in the product form of $g$. We give an example of an application of this alternative GeometricXL algorithm in Appendix A

\section{A Rank-2 Product Polynomial in Even Characteristic}

We now suppose in this and subsequent sections that the field $\mathbb{F}$ is of even characteristic, and we wish to find solutions to $f_{1}=\ldots=f_{m}=0$, where $f_{1}, \ldots, f_{m}$ are homogeneous polynomials of degree $D$ in $\mathbb{F}\left[x_{1}, \ldots, x_{m}\right]$. For a GeometricXL algorithm in even characteristic, we wish to find a linear combination of $f_{1}, \ldots, f_{m}$ that is a rank-2 product polynomial, that is we wish to find $g$ such that

$$
g=\sum_{l=1}^{m} \lambda_{l} f_{l}=\prod_{i=1}^{D}\left(\theta_{i} L+\theta_{i}^{\prime} L^{\prime}\right)=\sum_{i=0}^{D} \alpha_{i}^{2} L^{i}\left(L^{\prime}\right)^{D-i},
$$

for some $\alpha_{i} \in \mathbb{F}$ (as $\mathbb{F}$ has even characteristic), $\theta_{i}, \theta_{i}^{\prime} \in \overline{\mathbb{F}}$ (some extension field of $\mathbb{F}$ ) and homogeneous linear polynomials $L, L^{\prime} \in \mathbb{F}\left[x_{0}, \ldots, x_{n}\right]$. These homogeneous linear polynomials $L$ and $L^{\prime}$ can be written as

$$
L=\sum_{j=0}^{n} a_{j} x_{j} \text { and } L^{\prime}=\sum_{j=0}^{n} a_{j}^{\prime} x_{j} .
$$

The alternative description of the GeometricXL algorithm given in Section 2.2 finds a rank-2 product polynomial by finding a first order partial derivatives matrix of rank 2 in the case that the field characteristic $p$ satisfies $p=0$ or $p>D$. However, this property still holds in even characteristic, though the converse is not true, as the irreducible polynomial $x_{0}^{2}+x_{1} x_{2}$ over $\mathrm{GF}(2)$ with a partial derivatives matrix of rank 2 demonstrates.

We now discuss the properties of the partial derivatives matrix of a rank-2 product polynomial in even characteristic. We let $W_{D}$ denote the vector space of homogeneous polynomials over $\mathbb{F}$ of degree $D$ in $n+1$ variables, so $W_{D}$ has dimension $\left(\begin{array}{c}n+D \\ D\end{array}\right)[9]$. In the terminology of [12, $W_{D}$ is $\mathbb{S}^{D}\left(V^{*}\right)$, the $D^{t h}$ symmetric power of the dual space $V^{*}$ of the standard vector space $V$ of dimension $n+1$ over $\mathbb{F}$. We now define certain subspaces of $W_{D}$ that we consider in the development of a GeometricXL algorithm for even characteristic. 
Definition 3. The $\mathcal{L}^{2} \mathcal{S}$-subspace of $W_{D}$ for even degree $D=2 s+2$ is the subspace $U_{s}=\left\langle x_{i} x_{j} \mathbf{x}^{2} \mid \mathbf{x} \in W_{s}\right\rangle<W_{2 s+2}$.

Definition 4. The $\mathcal{L}^{1} \mathcal{S}$-subspace of $W_{D}$ for odd degree $D=2 s+1$ is the subspace $U_{s}^{\prime}=\left\langle x_{i} \mathbf{x}^{2} \mid \mathbf{x} \in W_{s}\right\rangle<W_{2 s+1}$.

Definition 5. The $\mathcal{L}^{0} \mathcal{S}$-subspace of $W_{D}$ for even degree $D=2 s$ is the subspace $U_{s}^{\prime \prime}=\left\langle\mathbf{x}^{2} \mid \mathbf{x} \in W_{s}\right\rangle<W_{2 s}$.

Lemma 5. Any $\mathcal{L}^{2} \mathcal{S}$-subspace $U_{s}, \mathcal{L}^{1} \mathcal{S}$-subspace $U_{s}^{\prime}$ and $\mathcal{L}^{0} \mathcal{S}$-subspace $U_{s}^{\prime \prime}$ is invariant under collineation. The dimensions of these subspaces are given by:

$$
\begin{aligned}
\operatorname{Dim}\left(U_{s}\right) & =\left(\begin{array}{c}
n+1 \\
2
\end{array}\right)\left(\begin{array}{c}
n+s \\
s
\end{array}\right)+\left(\begin{array}{c}
n+s+1 \\
s+1
\end{array}\right), \\
\operatorname{Dim}\left(U_{s}^{\prime}\right) & =\left(\begin{array}{c}
n+1 \\
1
\end{array}\right)\left(\begin{array}{c}
n+s \\
s
\end{array}\right), \\
\operatorname{Dim}\left(U_{s}^{\prime \prime}\right) & =\left(\begin{array}{c}
n+s \\
s
\end{array}\right) .
\end{aligned}
$$

The partial derivative mapping is a linear mapping, so any series of partial derivatives defines linear transformations $W_{2 s+2} \rightarrow W_{2 s+1} \rightarrow W_{2 s} \rightarrow W_{2 s-1}$. Thus any series of repeated partial differentiation in even characteristic gives rise to a series of linear transformations $U_{s} \rightarrow U_{s}^{\prime} \rightarrow U_{s}^{\prime \prime} \rightarrow\{0\}$ or informally $\mathcal{L}^{2} \mathcal{S} \rightarrow \mathcal{L}^{1} \mathcal{S} \rightarrow \mathcal{L}^{0} \mathcal{S} \rightarrow 0$. Our analysis of rank-2 product polynomials in even characteristic now proceeds by considering even and odd degree polynomials as separate cases.

\subsection{A Rank-2 Product Polynomial of Even Degree}

We can write the even degree $D$ as $D=2 s+2$, so a rank-2 product polynomial $g \in \mathcal{R}_{\mathbb{F}, n}^{2 s+2}$ can be expressed as

$g=\sum_{i=0}^{2 s+2} \alpha_{i}^{2} L^{i}\left(L^{\prime}\right)^{2 s+2-i}=\sum_{i=0}^{s+1} \alpha_{2 i}^{2} L^{2 i}\left(L^{\prime}\right)^{2(s+1-i)}+\sum_{i=0}^{s} \alpha_{2 i+1}^{2} L^{2 i+1}\left(L^{\prime}\right)^{2 s+1-2 i}$.

Thus we can express a rank- 2 product polynomial $g$ of even degree as

$$
g=\left(\sum_{i=0}^{s+1} \alpha_{2 i} L^{i}\left(L^{\prime}\right)^{(s+1-i)}\right)^{2}+L L^{\prime}\left(\sum_{i=0}^{s} \alpha_{2 i+1} L^{i}\left(L^{\prime}\right)^{s-i}\right)^{2} .
$$

We denote the first square of degree $2(s+1)$ by $S^{*}$ and the second square of degree $2 s$ by $S$, so $g$ is given by

$$
g=L L^{\prime} S+S^{*} .
$$

As $\mathbb{F}$ has even characteristic, any partial derivative of $S$ or $S^{*}$ vanishes, so a partial derivative of $g \in \mathcal{R}_{\mathbb{F}, n}^{2 s+2}$ is given by

$$
\frac{\partial g}{\partial x_{l}}=\frac{\partial\left(L L^{\prime}\right)}{\partial x_{l}} S=\frac{\partial L}{\partial x_{l}} L^{\prime} S+\frac{\partial L^{\prime}}{\partial x_{l}} L S=a_{l}\left(L^{\prime} S\right)+a_{l}^{\prime}(L S) .
$$

We have therefore shown that the partial derivative of a rank-2 product polynomial of even degree with respect to any variable is a linear combination of $L^{\prime} S$ and $L S$. The above comments are summarised in Lemma 6 . 
Lemma 6. Let $g \in \mathbb{F}\left[x_{0}, \ldots, x_{n}\right]$ be a rank-2 product polynomial of even degree $2 s+2$. If the field $\mathbb{F}$ has even characteristic, then $g \in \mathcal{R}_{\mathbb{F}, n}^{2 s+2}$ has the following properties:

1. $g \in U_{s}$, the $\mathcal{L}^{2} \mathcal{S}$-subspace;

2. $\frac{\partial g}{\partial x_{l}} \in U_{s}^{\prime}$, the $\mathcal{L}^{1} \mathcal{S}$-subspace;

3. the partial derivatives matrix $C_{g}^{(1)}$ has rank at most 2 .

\subsection{A Rank-2 Product Polynomial of Odd Degree}

We can write the odd degree $D$ as $D=2 s+1$, so a rank-2 product polynomial $g \in \mathcal{R}_{\mathbb{F}, n}^{2 s+1}$ can be expressed as

$$
g=\sum_{i=0}^{2 s+1} \alpha_{i}^{2} L^{i}\left(L^{\prime}\right)^{2 s+1-i}=\sum_{i=0}^{s} \alpha_{2 i}^{2} L^{2 i}\left(L^{\prime}\right)^{2 s+1-2 i}+\sum_{i=0}^{s} \alpha_{2 i+1}^{2} L^{2 i+1}\left(L^{\prime}\right)^{2 s-2 i} .
$$

We can thus express a rank-2 product polynomial $g$ of odd degree as

$$
g=L^{\prime}\left(\sum_{i=0}^{s} \alpha_{2 i} L^{i} L^{\prime s-i}\right)^{2}+L\left(\sum_{i=0}^{s} \alpha_{2 i+1} L^{i} L^{\prime s-i}\right)^{2}
$$

The above expression for $g$ consists of two squares of degree $2 s$, which we denote by $S^{\prime}$ and $S$ respectively. We can thus express $g$ as

$$
g=L S+L^{\prime} S^{\prime} .
$$

As $S$ and $S^{\prime}$ are square polynomials over a field of even characteristic, any partial derivative of $S$ or $S^{\prime}$ is zero. Thus a partial derivative of $g \in \mathcal{R}_{\mathbb{F}, n}^{2 s+1}$ is given by

$$
\frac{\partial g}{\partial x_{l}}=\frac{\partial L}{\partial x_{l}} S+\frac{\partial L^{\prime}}{\partial x_{l}} S^{\prime}=a_{l} S+a_{l}^{\prime} S^{\prime}
$$

We have therefore shown that the partial derivative of a rank-2 product polynomial of odd degree with respect to any variable is a linear combination of $S$ and $S^{\prime}$. The above comments are summarised by Lemma 7

Lemma 7. Let $g \in \mathbb{F}\left[x_{0}, \ldots, x_{n}\right]$ be a rank-2 product polynomial of odd degree $2 s+1$. If the field $\mathbb{F}$ has even characteristic, then $g \in \mathcal{R}_{\mathbb{F}, n}^{2 s+1}$ has the following properties:

1. $g \in U_{s}^{\prime}$, the $\mathcal{L}^{1} \mathcal{S}$-subspace,

2. $\frac{\partial g}{\partial x_{l}} \in U_{s}^{\prime \prime}$, that is any partial derivative of $g$ is a square;

3. the partial derivatives matrix $C_{g}^{(1)}$ has rank at most 2 . 


\subsection{A Necessary Criterion for a Rank-2 Product Polynomial}

Definition 6. The $\mathcal{L S}$-criterion for a homogeneous multivariate polynomial $g$ is that $g$ is an element either of the $\mathcal{L}^{2} \mathcal{S}$-subspace (even degree) or the $\mathcal{L}^{1} \mathcal{S}$ subspace (odd degree).

Lemmas 6 and 7 show that for a polynomial $g=\sum_{l=1}^{m} \lambda_{l} f_{l}$ to be a rank-2 product polynomial, $g$ has to satisfy the $\mathcal{L S}$-criterion. For equation systems of cubic or higher degree, Lemma [5] shows that the dimension of the $\mathcal{L}^{2} \mathcal{S}$-subspace or the $\mathcal{L}^{1} \mathcal{S}$-subspace is generally far smaller than the dimension of $W_{D}$. For such equation systems, we can therefore obtain many linear constraints on $\lambda_{1}, \ldots, \lambda_{m}$ for $g=\sum_{l=1}^{m} \lambda_{l} f_{l}$ to be a rank-2 product polynomial. These linear constraints can be processed very efficiently using basic linear algebra. Thus this criterion alone can easily greatly reduce the size of or even solve the equation system.

We give an example of solving a cubic system over a field of even characteristic by considering membership of the $\mathcal{L}^{1} \mathcal{S}$-subspace $U_{1}^{\prime}$ in Appendix B. However, both the $\mathcal{L}^{1} \mathcal{S}$-subspace and and $\mathcal{L}^{2} \mathcal{S}$-subspace contain many polynomials that are not rank-2 product polynomials, so there may be a requirement for further processing after this preliminary linear filtering. Furthermore, this criterion cannot be applied to quadratic systems as $U_{0}=W_{2}$. We discuss further techniques to identify rank-2 product polynomials in Section 4 .

\section{Identification of a Rank-2 Product Polynomial}

The basic idea of the GeometricXL algorithm to solve the homogeneous system $f_{1}=\ldots=f_{m}=0$ of degree $D$ in $n+1$ variables is to find a linear combination such that $g=\sum_{l=1}^{m} \lambda_{l} f_{l}$ is a rank-2 product polynomial, that is $g \in \mathcal{R}_{\mathbb{F}, n}^{D}$. However, in even characteristic we can use the properties of rank-2 product polynomials in even characteristic given by Lemma 6 and Lemma 7 to help identify such polynomials. This should enable us subsequently to develop a method for fields of even characteristic based on the GeometricXL algorithm. However, we note that such an algorithm still has the potential problem discussed in Section 7.5 of [12, namely the possibility of nested multiple linear factors only one of which corresponds to a solution.

\subsection{Multivariate Quadratic Systems}

We consider a field $\mathbb{F}$ of even characteristic and a homogeneous quadratic equation system $f_{1}=\ldots=f_{m}=0$ over $\mathbb{F}$. We need to find a linear combination $g=\sum_{l=1}^{m} \lambda_{l} f_{l}$ such that $g$ is a rank-2 product polynomial of degree 2. However, any such $g \in \mathcal{R}_{\mathbb{F}, n}^{2}$ can be regarded as the product of the two homogeneous linear polynomials $L$ and $L^{\prime}$ (Section 3.1), that is $g$ is of the form

$$
\left(a_{0} x_{0}+\ldots+a_{0} x_{0}\right)\left(a_{0}^{\prime} x_{0}+\ldots+a_{n}^{\prime} x_{n}\right)=\sum_{i=0}^{n} a_{i} a_{i}^{\prime} x_{i}^{2}+\sum_{i=1}^{n} \sum_{j=0}^{i-1}\left(a_{i} a_{j}^{\prime}+a_{i}^{\prime} a_{j}\right) x_{i} x_{j} .
$$


We can write $\Delta_{i j}=a_{i} a_{j}^{\prime}+a_{j} a_{i}^{\prime}$, so the product of two homogeneous linear polynomials can be expressed as

$$
g=L L^{\prime}=\left(a_{0} x_{0}+\ldots a_{n} x_{n}\right)\left(a_{0}^{\prime} x_{0}+\ldots a_{n}^{\prime} x_{n}\right)=\sum_{i=0}^{n} a_{i} a_{i}^{\prime} x_{i}^{2}+\sum_{i=1}^{n} \sum_{j=0}^{i-1} \Delta_{i j} x_{i} x_{j} .
$$

We can write the homogeneous quadratic polynomial $f_{l}(1 \leq l \leq m)$ as

$$
f_{l}=\sum_{i=0}^{n} d_{i i}^{(l)} x_{i}^{2}+\sum_{i=1}^{n} \sum_{j=0}^{i-1} d_{i j}^{(l)} x_{i} x_{j}
$$

for coefficients $d_{i j}^{(l)}$, so a rank-2 product polynomial $g=\sum_{l=1}^{m} \lambda_{l} f_{l}$ satisfies

$$
\begin{aligned}
& g=\sum_{i=0}^{n}\left(\sum_{l=1}^{m} \lambda_{l} d_{i i}^{(l)}\right) x_{i}^{2}+\sum_{i=1}^{n} \sum_{j=0}^{i-1}\left(\sum_{l=1}^{m} \lambda_{l} d_{i j}^{(l)}\right) x_{i} x_{j} \\
& =\sum_{i=0}^{n} \quad a_{i} a_{i}^{\prime} \quad x_{i}^{2}+\sum_{i=1}^{n} \sum_{j=0}^{i-1} \quad \Delta_{i j} \quad x_{i} x_{j} .
\end{aligned}
$$

Thus for $g$ to be a rank-2 product polynomial, we can see by equating coefficients of $x_{i} x_{j}(j<i)$ that we require $\lambda_{1}, \ldots, \lambda_{m}$ such that $\Delta_{i j}=\sum_{l=1}^{m} d_{i j}^{(l)} \lambda_{l}$.

Let $A$ be the $2 \times(n+1)$ matrix with rows given by the (unknown) coefficients of the linear polynomials $L$ and $L^{\prime}$, so

$$
A=\left(\begin{array}{ccccccc}
a_{0} & \ldots & a_{i} & \ldots & a_{j} & \ldots & a_{n} \\
a_{0}^{\prime} & \ldots & a_{i}^{\prime} & \ldots & a_{j}^{\prime} & \ldots & a_{n}^{\prime}
\end{array}\right),
$$

then the $\Delta_{i j}$ are the 2-minors $\left(2 \times 2\right.$ sub-determinants) of $A$. Now, there are $\left(\begin{array}{c}n+1 \\ 4\end{array}\right)$ 4 -minors of the $4 \times(n+1)$ matrix $\bar{A}=\left(\frac{A}{A}\right)$, and these are given by

$$
\mathcal{A}_{i_{1}, i_{2}, i_{3}, i_{4}}=\Delta_{i_{1}, i_{2}} \Delta_{i_{3}, i_{4}}+\Delta_{i_{1}, i_{3}} \Delta_{i_{2}, i_{4}}+\Delta_{i_{1}, i_{4}} \Delta_{i_{2}, i_{3}} .
$$

However, the matrix $\bar{A}$ clearly has rank at most 2, so every 4-minor of $\bar{A}$ is identically 0 . Thus we obtain $\left(\begin{array}{c}n+1 \\ 4\end{array}\right)$ identities $\mathcal{A}_{i_{1}, i_{2}, i_{3}, i_{4}}=0$. As each 4-minor $\mathcal{A}_{i_{1}, i_{2}, i_{3}, i_{4}}$ of $\bar{A}$ gives rise to a homogeneous quadratic expression in $\Delta_{i j}$, so each 4-minor identity $\mathcal{A}_{i_{1}, i_{2}, i_{3}, i_{4}}=0$ gives a homogeneous quadratic identity in $\Delta_{i j}$.

We saw above that for $g=\sum_{l=1}^{m} \lambda_{l} f_{l}$ to be a rank-2 product polynomial, we require that $\Delta_{i j}=\sum_{l=1}^{m} d_{i j}^{(l)} \lambda_{l}$. Thus the $\left(\begin{array}{c}n+1 \\ 4\end{array}\right)$ identities $\mathcal{A}_{i_{1}, i_{2}, i_{3}, i_{4}}=0$ give rise to a homogeneous quadratic system with $\left(\begin{array}{c}n+1 \\ 4\end{array}\right) \sim \frac{1}{24} n^{4}$ equations satisfied by $\lambda_{1}, \ldots, \lambda_{m}$. We can potentially solve this system using linearisation or some other simple technique. If there is a unique solution to this quadratic system in $\lambda_{1}, \ldots, \lambda_{m}$, then this can directly determine the solution of the original equation system. More generally, an analysis of this quadratic system gives much information about the original quadratic system, which could then be used with other techniques to provide a solution to the original system.

We make some comments about geometric aspects of this process in Section 5.2, and demonstrate the use of this process in finding a solution to a quadratic system in Appendix $\mathrm{C}$. 


\subsection{Multivariate Systems of Quartic or Higher Even Degree}

We consider a field $\mathbb{F}$ of even characteristic and a homogeneous equation system $f_{1}=\ldots=f_{m^{\prime}}=0$ of degree $D=2 s+2(s>0)$ over $\mathbb{F}$, where without loss of generality this equation system may have been obtained by applying the $\mathcal{L}^{2} \mathcal{S}$ criterion of Section 3.3 to a larger system $f_{1}=\ldots=f_{m}=0\left(\right.$ so $\left.m^{\prime} \leq m\right)$. We need to find a linear combination $g=\sum_{l=1}^{m^{\prime}} \lambda_{l} f_{l}$ such that $g$ is a rank-2 product polynomial, that is $g \in \mathcal{R}_{\mathbb{F}, n}^{2 s+2}$. In the quadratic case $(s=0)$, we consider the coefficients of the non-square monomials $x_{i} x_{j}$ (so $i \neq j$ ) to give conditions for a rank-2 product polynomial (Section 4.1). In the case of quartic and higher even degree, we first consider the coefficients $\Gamma_{i j}$ of $x_{i}^{2 s+1} x_{j}$ (for $i \neq j$ ) in $g$, a natural generalisation of this idea.

If $g$ is a rank-2 product polynomial of degree $2 s+2$, so $g \in \mathcal{R}_{\mathbb{F}, n}^{2 s+2}$, then $g=L L^{\prime} S+S^{*}$, where $L$ and $L^{\prime}$ are the homogeneous linear polynomials of Section 3 and $S$ and $S^{*}$ are homogeneous square polynomials of degree $2 s$ and $2 s+2$ respectively (Section 3.1). Thus if we let $s_{i}$ denote the coefficient of $x_{i}^{2 s}$ in $S$ we have

$$
\begin{aligned}
g & =\left(a_{i} x_{i}+a_{j} x_{j}+\ldots\right)\left(a_{i}^{\prime} x_{i}+a_{j}^{\prime} x_{j}+\ldots\right)\left(s_{i} x_{i}^{2 s}+\ldots\right)+S^{*} \\
& =\Gamma_{i j} x_{i}^{2 s+1} x_{j}+\ldots=s_{i} \Delta_{i j} x_{i}^{2 s+1} x_{j}+\ldots=\left(b_{i} a_{j}^{\prime}+b_{i}^{\prime} a_{j}\right) x_{i}^{2 s+1} x_{j}+\ldots,
\end{aligned}
$$

where $\Delta_{i j}=a_{i} a_{j}^{\prime}+a_{i}^{\prime} a_{j}$ is a 2-minor of the matrix $A$ of Section 4.1 and $b_{i}=s_{i} a_{i}$ and $b_{i}^{\prime}=s_{i} a_{i}^{\prime}$. Thus we have $\Gamma_{i j}=s_{i} \Delta_{i j}=b_{i} a_{j}^{\prime}+b_{i}^{\prime} a_{j}$ for $i \neq j$. For completeness, we set $\Gamma_{i i}=b_{i} a_{i}^{\prime}+b_{i}^{\prime} a_{i}=s_{i} a_{i} a_{i}^{\prime}+s_{i} a_{i} a_{i}^{\prime}=0$. The matrix $\Gamma=\left(\Gamma_{i j}\right)$ can be expressed as $\Gamma=B+B^{\prime}$, where $B=\mathbf{b a}^{\prime T}$ and $B^{\prime}=\mathbf{b}^{\prime} \mathbf{a}^{T}$ for appropriate column vectors of coefficients $\mathbf{b}, \mathbf{a}, \mathbf{b}^{\prime}$ and $\mathbf{a}^{\prime}$, so $B$ and $B^{\prime}$ are both matrices of rank 1. Thus the matrix $\Gamma$ of coefficients of $x_{i}^{2 s+1} x_{j}$ (for $i \neq j$ with $\Gamma_{i i}=0$ ) of a rank-2 product polynomial has rank at most 2 as it is the sum $\Gamma=B+B^{\prime}$ of two matrices of rank 1 . There are $\left(\begin{array}{c}n+1 \\ 3\end{array}\right)^{2} 3$-minors of $\Gamma$, and they are given by

$$
\begin{aligned}
\mathcal{B}_{i_{1}, i_{2}, i_{3}, j_{1}, j_{2}, j_{3}}= & \Gamma_{i_{1}, j_{1}} \Gamma_{i_{2}, j_{2}} \Gamma_{i_{3}, j_{3}}+\Gamma_{i_{1}, j_{1}} \Gamma_{i_{2}, j_{3}} \Gamma_{i_{3}, j_{2}}+\Gamma_{i_{2}, j_{1}} \Gamma_{i_{1}, j_{2}} \Gamma_{i_{3}, j_{3}} \\
& +\Gamma_{i_{2}, j_{1}} \Gamma_{i_{1}, j_{3}} \Gamma_{i_{3}, j_{2}}+\Gamma_{i_{3}, j_{1}} \Gamma_{i_{1}, j_{2}} \Gamma_{i_{2}, j_{3}}+\Gamma_{i_{3}, j_{1}} \Gamma_{i_{1}, j_{3}} \Gamma_{i_{2}, j_{2}} .
\end{aligned}
$$

However, every 3 -minor of the matrix $\Gamma$ of rank 2 vanishes, so we obtain $\left(\begin{array}{c}n+1 \\ 3\end{array}\right)^{2}$ identities $\mathcal{B}_{i_{1}, i_{2}, i_{3}, j_{1}, j_{2}, j_{3}}=0$.

For $g$ to be a rank-2 product polynomial, we can see by equating coefficients of $x_{i}^{2 s+1} x_{j}(i \neq j)$ that we require $\lambda_{1}, \ldots, \lambda_{m^{\prime}}$ such that $\Delta_{i j}=\sum_{l=1}^{m^{\prime}} d_{i j}^{(l)} \lambda_{l}$, where $d_{i j}^{(l)}$ denote the coefficient of $x_{i}^{2 s+1} x_{j}$ in $f_{l}$. Thus the $\left(\begin{array}{c}n+1 \\ 3\end{array}\right)^{2}$ identities $\mathcal{B}_{i_{1}, i_{2}, i_{3}, j_{1}, j_{2}, j_{3}}=0$ give rise to a homogeneous cubic system with $\left(\begin{array}{c}n+1 \\ 3\end{array}\right)^{2} \sim \frac{1}{36} n^{6}$ equations satisfied by $\lambda_{1}, \ldots, \lambda_{m^{\prime}}$. It may now be possible to solve this resulting cubic system in $\lambda_{1}, \ldots, \lambda_{m^{\prime}}$ by linearisation or some other method, so providing a solution to the original equation system. We provide an example of this process to solve a homogeneous quartic system in Appendix D.

We also note that it is very easy to produce further homogeneous cubic equations in $\lambda_{1}, \ldots, \lambda_{m^{\prime}}$ if required, as $\Gamma$ is far from being the only matrix of coefficients having rank 2 . For example, a similar argument to that given above for $\Gamma$ shows that the matrix of coefficients of $x_{0}^{2} x_{i}^{2 s-1} x_{j}$ also has rank 2 and so on. 


\subsection{Multivariate Systems of Odd Degree}

We consider a field $\mathbb{F}$ of even characteristic and a homogeneous equation system $f_{1}=\ldots=f_{m^{\prime}}=0$ of odd degree $D=2 s+1$ over $\mathbb{F}$, where without loss of generality this equation system may have been obtained by applying the $\mathcal{L}^{1} \mathcal{S}$ criterion of Section 3.3 to a larger system $f_{1}=\ldots=f_{m}=0\left(\right.$ so $\left.m^{\prime} \leq m\right)$. We need to find a linear combination $g=\sum_{l=1}^{m^{\prime}} \lambda_{l} f_{l}$ such that $g$ is a rank-2 product polynomial, that is $g \in \mathcal{R}_{\mathbb{F}, n}^{2 s+1}$. In a similar way to the case for even degree, we consider the coefficients $\Lambda_{i j}$ of $x_{i}^{2 s} x_{j}$ in $g$, including the coefficients $\Lambda_{i i}$ of $x_{i}^{2 s+1}$.

If $g$ is a rank-2 product polynomial of degree $2 s+1$, so $g \in \mathcal{R}_{\mathbb{F}, n}^{2 s+1}$, then we can express $g$ as $g=L S+L^{\prime} S^{\prime}$, where $L$ and $L^{\prime}$ are homogeneous linear polynomials and $S$ and $S^{\prime}$ are homogeneous square polynomials of degree $2 s$ (Section 3.2 ). We can thus express a summand of $g$ as

$$
L S=c_{i j} x_{i}^{2 s} x_{j}+\ldots=\left(s_{i} x_{i}^{2 s}+\ldots\right)\left(a_{j} x_{j}+\ldots\right)=s_{i} a_{j} x_{i}^{2 s} x_{j}+\ldots,
$$

so the matrix $C=\left(c_{i j}\right)$ has rank 1 as $C=\mathbf{s a}^{T}$ for appropriate column vectors of coefficients $\mathbf{s}$ and $\mathbf{a}$. This means we can express $g$ as

$$
g=\Lambda_{i j} x_{i}^{2 s} x_{j}+\ldots=L S+L^{\prime} S^{\prime}=\left(c_{i j}+c_{i j}^{\prime}\right) x_{i}^{2 s} x_{j}+\ldots
$$

Thus the matrix $\Lambda=\left(\Lambda_{i j}\right)$ of coefficients of $x_{i}^{2 s} x_{j}$ of a rank-2 product polynomial has rank at most 2 as it is the sum $\Lambda=C+C^{\prime}$ of two matrices of rank 1 . There are $\left(\begin{array}{c}n+1 \\ 3\end{array}\right)^{2} 3$-minors of $\Lambda$, and they are given by

$$
\begin{aligned}
\mathcal{C}_{i_{1}, i_{2}, i_{3}, j_{1}, j_{2}, j_{3}}= & \Lambda_{i_{1}, j_{1}} \Lambda_{i_{2}, j_{2}} \Lambda_{i_{3}, j_{3}}+\Lambda_{i_{1}, j_{1}} \Lambda_{i_{2}, j_{3}} \Lambda_{i_{3}, j_{2}}+\Lambda_{i_{2}, j_{1}} \Lambda_{i_{1}, j_{2}} \Lambda_{i_{3}, j_{3}} \\
& +\Lambda_{i_{2}, j_{1}} \Lambda_{i_{1}, j_{3}} \Lambda_{i_{3}, j_{2}}+\Lambda_{i_{3}, j_{1}} \Lambda_{i_{1}, j_{2}} \Lambda_{i_{2}, j_{3}}+\Lambda_{i_{3}, j_{1}} \Lambda_{i_{1}, j_{3}} \Lambda_{i_{2}, j_{2}}
\end{aligned}
$$

However, every 3 -minor of $\Lambda$ vanishes as $\Lambda$ has rank 2 , so we obtain $\left(\begin{array}{c}n+1 \\ 3\end{array}\right)^{2}$ identities $\mathcal{C}_{i_{1}, i_{2}, i_{3}, j_{1}, j_{2}, j_{3}}=0$.

For $g$ to be a rank-2 product polynomial, we can see by equating coefficients of $x_{i}^{2 s} x_{j}(i \neq j)$ that we require $\lambda_{1}, \ldots, \lambda_{m^{\prime}}$ such that $\Delta_{i j}=\sum_{l=1}^{m^{\prime}} d_{i j}^{(l)} \lambda_{l}$, where $d_{i j}^{(l)}$ denote the coefficient of $x_{i}^{2 s} x_{j}$ in $f_{l}$. Thus the $\left(\begin{array}{c}n+1 \\ 3\end{array}\right)^{2}$ identities $\mathcal{C}_{i_{1}, i_{2}, i_{3}, j_{1}, j_{2}, j_{3}}=0$ give rise to a homogeneous cubic system with $\left(\begin{array}{c}n+1 \\ 3\end{array}\right)^{2} \sim \frac{1}{36} n^{6}$ equations satisfied by $\lambda_{1}, \ldots, \lambda_{m^{\prime}}$. It may now be possible to solve this resulting cubic system in $\lambda_{1}, \ldots, \lambda_{m^{\prime}}$ by linearisation or some other method, so providing a solution to the original equation system. We provide an example of this process to solve a quintic system in Appendix E. Furthermore, as in Section 4.2, we note that is very easy to produce further homogeneous cubic equations in $\lambda_{1}, \ldots, \lambda_{m^{\prime}}$ if required as there are many other coefficient matrices having rank 2 , for example the matrix of coefficients of $x_{0}^{2} x_{i}^{2 s-2} x_{j}$.

\section{A Geometrical Interpretation}

The geometric techniques of Section 4 can be interpreted using the Grassmannian variety 3910. We give some basic properties of the Grassmannian variety in Section 5.1 and discuss their application in Section 5.2 . 


\subsection{The Grassmannian Variety and Exterior Algebra}

We recall that $W_{1}$ denotes the vector space of homogeneous linear polynomials over $\mathbb{F}$ of degree $D$ in $n+1$ variables (Section 3 ). Thus the two linear polynomials $L$ and $L^{\prime}$ at the heart of Definition 1 are elements of $W_{1}$, so the pair $\left(L, L^{\prime}\right)$ defines a (projective) line in the projective space $\mathcal{P}\left(W_{1}\right)$. The Grassmannian $G r_{2}\left(W_{1}\right)$ is the set of all 2-dimensional subspaces of $W_{1}$ or equivalently the set of all projective lines in this projective space $\mathcal{P}\left(W_{1}\right)$ [910.

The tensor product $W_{1} \otimes W_{1}$ is the $(n+1)^{2}$-dimensional vector space with basis vectors the set of formal symbols $x_{i} \otimes x_{j}$ and a bilinear inclusion map from $W_{1} \times W_{1}$ to $W_{1} \otimes W_{1}$ 4]12. The symmetric square $\mathbb{S}^{2}\left(W_{1}\right)$ is the subspace of all symmetric tensors $\left(\left\{t_{i j} \in W_{1} \otimes W_{1} \mid t_{i j}=t_{j i}\right\}\right)$, a subspace of dimension $\frac{1}{2}(n+$ 1) $(n+2)$ 9[12. In even characteristic, the symmetric square can be decomposed as

$$
\mathbb{S}^{2}\left(W_{1}\right) \cong\left\langle\left(x_{i} \otimes x_{i}\right)_{i}\right\rangle \oplus \frac{\mathbb{S}^{2}\left(W_{1}\right)}{\left\langle\left(x_{i} \otimes x_{i}\right)_{i}\right\rangle},
$$

where the second (quotient) summand is the degree-2 part of the exterior algebra, a space of dimension $\frac{1}{2} n(n+1)$, which we denote by $\mathbb{E}^{2}\left(W_{1}\right)$. Thus we have $\mathbb{S}^{2}\left(W_{1}\right) \cong\left\langle\left(x_{i} \otimes x_{i}\right)_{i}\right\rangle \oplus \mathbb{E}^{2}\left(W_{1}\right)$. The Grassmannian or Plücker embedding of the Grassmannian $G_{2}\left(W_{1}\right)$ in the degree-2 part of the exterior algebra is an injective mapping $\psi: G r_{2}\left(W_{1}\right) \rightarrow \mathcal{P}\left(\mathbb{E}^{2}\left(W_{1}\right)\right)$ defined by $\left(L, L^{\prime}\right) \mapsto L \wedge L^{\prime}$ or equivalently

$$
\left(L, L^{\prime}\right)=\left(\sum_{i=0}^{n} a_{i} x_{i}, \sum_{i=0}^{n} a_{i}^{\prime} x_{i}\right) \mapsto \sum_{i=0}^{n} \sum_{j=0}^{i-1}\left(a_{i} a_{j}^{\prime}+a_{i}^{\prime} a_{j}\right)\left(x_{i} \wedge x_{j}\right) .
$$

This vector of co-ordinates $\left(a_{i} a_{j}+a_{i}^{\prime} a_{j}\right)$ is known as the Grassmannian or Plücker co-ordinates of the (projective) line defined by $L$ and $L^{\prime}$. Thus the Grassmannian co-ordinates of the line defined by $L$ and $L^{\prime}$ are given by the 2-minors of the matrix $A$ of Section 4.1, with rows given by $L$ and $L^{\prime}$.

The Grassmannian embedding allows the representation of (projective) lines in $\mathcal{P}\left(W_{1}\right)$ as distinct points in the projective space $\mathcal{P}\left(\mathbb{E}^{2}\left(W_{1}\right)\right)$, which is isomorphic to $\mathrm{PG}\left(\frac{1}{2} n(n+1)-1, \mathbb{F}\right)$. The Grassmannian variety $\mathcal{G}$ of $\mathcal{P}\left(\mathbb{E}^{2}\left(W_{1}\right)\right)$ is the set of all such embedded lines [3]. Thus the Grassmannian variety $\mathcal{G}$ is simply $\psi\left(G r_{2}\left(W_{1}\right)\right)$, the image of the Grassmannian $G r_{2}\left(W_{1}\right)$, so $\mathcal{G}$ is defined by

$$
\mathcal{G}=\left\{\left\langle\left(\Delta_{21}, \Delta_{31}, \ldots, \Delta_{n+1, n-1}, \Delta_{n+1, n}\right)^{T}\right\rangle \in \mathcal{P}\left(\mathbb{E}^{2}\left(W_{1}\right)\right) \mid \mathcal{A}_{i_{1}, i_{2}, i_{3}, i_{4}}=0\right\},
$$

where $\mathcal{A}_{i_{1}, i_{2}, i_{3}, i_{4}}$ is the quadratic identity of Section 4.1. Thus the Grassmannian variety $\mathcal{G}$ can be defined by the intersection of quadrics in $\mathcal{P}\left(\mathbb{E}^{2}\left(W_{1}\right)\right)$.

\subsection{The Grassmannian Variety and the GeometricXL Algorithm}

We consider first a GeometricXL algorithm for homogeneous quadratic systems in even characteristic, discussed in Section 4.1. We need to find $g=\sum_{i=1}^{m} \lambda_{l} f_{l}$ such that $g$ is the product of two homogeneous linear polynomials $L$ and $L^{\prime}$. We 
can think of $g$ in the natural and obvious way as an element of $\mathbb{S}^{2}\left(W_{1}\right)$. Thus we can define the canonical projection $\pi(g)$ of $g$ onto the exterior algebra $\mathbb{E}^{2}\left(W_{1}\right)$, so $\pi(g)$ is the square-free part of $g$. However, $g$ is a product of linear polynomials if and only if $\pi(g) \in \mathcal{G}$, the Grassmannian variety. Thus the geometrical interpretation of the process of Section 4.1 is that it is a process that first attempts to find polynomials with no square terms in the variety

$$
\mathcal{G} \bigcap\left\langle\pi\left(f_{1}\right), \ldots, \pi\left(f_{m}\right)\right\rangle,
$$

and then analyses these polynomials to find rank-2 product polynomials.

There are some further obvious geometric comments that can be made about higher degree equations systems. For example, for odd degree systems any solution lies on the secant variety of the variety of all polynomials which are the product of a linear polynomial and a square polynomial, and such secant varieties are the basis of the GeometricXL algorithm when the characteristic exceeds $D$ or is zero [12. However, a full geometric interpretation is still needed for cubic and higher degree equation systems, and could provide interesting ideas for solution methods. Such an interpretation is very likely to depend on the Grassmannian variety $\mathcal{G}$ as any rank-2 product polynomial depends fundamentally on the linear polynomials $L$ and $L^{\prime}$, or equivalently such an interpretation depends fundamentally on a point of the Grassmannian variety $\mathcal{G}$.

\section{The EGHAM Process}

In the common cryptographic situation of attempting to find a unique solution to a homogeneous equation system $f_{1}=\ldots=f_{m}=0$ over a field of even characteristic, the goal of the XL algorithm is to find a bivariate polynomial $g=\sum_{i=1}^{m} \lambda_{i} f_{i}$, and the goal of the Gröbner basis algorithm (under lexicographic ordering) is to find such a bivariate polynomial $g$ in the reduced Gröbner basis. However, we have shown that any such linear combination of homogeneous polynomials generating such a bivariate polynomial $g$ must satisfy the $\mathcal{L S}$-criterion. Thus the $\mathcal{L S}$-criterion (Definition 6) can be applied as part of a standard Gröbner basis or XL algorithm in this situation. Applying the $\mathcal{L S}$-criterion generally greatly reduces the number of polynomials under consideration by an XL algorithm or Gröbner basis algorithm. Furthermore, the reduced set of equations obtained by applying the $\mathcal{L} \mathcal{S}$-criterion can be considered for any order of the variables, as the $\mathcal{L S}$-criterion is independent of the order of the variables. This means that the XL algorithm or Gröbner basis algorithm with the $\mathcal{L S}$-criterion is far more efficient at finding an appropriate bivariate polynomial or concluding that no such bivariate polynomial exists for a given degree (under any variable order).

We can therefore include the $\mathcal{L S}$-criterion as part of an adaptation, using the ideas of Section 4. of the GeometricXL algorithm [12] for use in fields of even characteristic. This gives us a process for implementing an even (characteristic) GeometricXL heuristic algorithmic method, which we term the EGHAM process and which we describe in Figure 1 . 
- Generate a homogeneous system $f_{1}=\ldots=f_{m}=0$ of degree $D$ from an original equation system in even characteristic.

- Apply the $\mathcal{L} \mathcal{S}$-criterion (Definition 6), that is consider only linear combinations of $f_{1}, \ldots, f_{m}$ in the $\mathcal{L}^{2} \mathcal{S}$-subspace or $\mathcal{L}^{1} \mathcal{S}$-subspace for systems of cubic or higher degrees, so generally reducing the size of the problem from $m$ equations to $m^{\prime}$ equations.

- Construct a quadratic or cubic equation system in the coefficients $\lambda_{1}, \ldots, \lambda_{m^{\prime}}$ for $\sum_{i=1}^{m^{\prime}} \lambda_{i} f_{i}$ to be a rank-2 product polynomial, for example by considering the matrix of coefficients of $x_{i}^{D-1} x_{j}$ (Section 4).

- Analyse this quadratic or cubic system, perhaps by linearisation, to find a rank-2 product polynomial in $\left\langle f_{1}, \ldots, f_{m^{\prime}}\right\rangle$, which can then be factored to provide information about the solution of (or even solve) $f_{1}=\ldots=f_{m}=0$.

- Apply this solution information directly to the original equation system.

Fig. 1. Basic Description of the EGHAM Process

The EGHAM process is an adaptation of the GeometricXL algorithm to fields of even characteristic, and so is a geometric generalisation of an XL algorithm for such fields. Thus the EGHAM process generates equations of at worst the same degree as either a Gröbner basis (with lexicographic ordering) or an XL algorithm, though usually by processing a far smaller equation system through the application of the $\mathcal{L} \mathcal{S}$-criterion. However, as is clearly demonstrated by the examples in the Appendices, the EGHAM process can use polynomials of a much smaller degree for many equation systems in even characteristic than a Gröbner basis algorithm or an XL algorithm.

\section{Conclusions}

A major contribution of this paper is the development of the $\mathcal{L} \mathcal{S}$-criterion (Definition [6) in the solution of homogeneous cryptographic equation systems in fields of even characteristic. We have used the $\mathcal{L} \mathcal{S}$-criterion to develop the EGHAM process, an adaptation of the GeometricXL algorithm [12] which is suitable for use in fields of even characteristic. The EGHAM process can find the solution of a cryptographic equation system in even characteristic much more efficiently than a standard Gröbner basis or XL algorithm in many cases.

\section{Acknowledgments}

We wish to thank Martin Albrecht and our referees for their comments.

\section{References}

1. Ars, G., Faugère, J.-C., Imai, H., Kawazoe, M., Sugita, M.: Comparison between XL and Gröbner Basis Algorithms. In: Lee, P.J. (ed.) ASIACRYPT 2004. LNCS, vol. 3329, pp. 338-353. Springer, Heidelberg (2004) 
2. Buchberger, B.: Ein Algorithmus zum Auffinden der Basiselemente des Restklassenringes nach einem nulldimensionalen Polynomideal. PhD thesis, Universität Innsbruck (1965)

3. Burau, W.: Mehrdimensionale Projecktive und Höhere Geometrie, Berlin (1961)

4. Cohn, P.: Classical Algebra. John Wiley, Chichester (2000)

5. Courtois, N., Klimov, A., Patarin, J., Shamir, A.: Efficient Algorithms for Solving Overdefined Systems of Multivariate Polynomial Equations. In: Preneel, B. (ed.) EUROCRYPT 2000. LNCS, vol. 1807, pp. 392-407. Springer, Heidelberg (2000)

6. Faugère, J.-C.: A New Efficient Algorithm for Computing Gröbner bases (F4). Journal of Pure and Applied Algebra 139, 61-88 (1999)

7. Faugère, J.-C.: A New Efficient Algorithm for Computing Gröbner Bases without Reduction to Zero (F5). In: Mora, T. (ed.) International Symposium on Symbolic and Algebraic Computation - ISSAC 2002, pp. 75-83 (2002)

8. Faugère, J.-C., Levy-dit-Vehel, F., Perret, L.: Cryptanalysis of MinRank. In: Wagner, D. (ed.) CRYPTO 2008. LNCS, vol. 5157, pp. 280-296. Springer, Heidelberg (2008)

9. Harris, J.: Algebraic Geometry: A First Course. Graduate Text in Mathematics, vol. 133. Springer, Heidelberg (1992)

10. Hirschfeld, J.W.P., Thas, J.A.: General Galois Geometries. Oxford University Press, Oxford (1991)

11. Iarrobino, A., Kanev, V.: Power Sums, Gorenstein Algebras and Determinantal Loci. Lecture Notes in Mathematics, vol. 1725. Springer, Heidelberg (1999)

12. Murphy, S., Paterson, M.B.: A Geometric View of Cryptographic Equation Solving. Journal of Mathematical Cryptology 2, 63-107 (2008)

\section{A The Alternative GeometricXL Algorithm}

We demonstrate the alternative GeometricXL algorithm (Section 2.2) on a homogeneous cubic system $f_{1}=f_{2}=f_{3}=0$ of three equations in three variables over GF $(37)$. We give the coefficents of $f_{1}, f_{2}, f_{3}$ below with respect to the lexicographic monomial ordering $x_{0}^{3}, x_{0}^{2} x_{1}, \ldots, x_{2}^{3}$.

$$
\begin{array}{rrrrrrrrrr}
23 & 27 & 15 & 25 & 11 & 24 & 26 & 7 & 21 & 36 \\
21 & 35 & 2 & 18 & 4 & 1 & 29 & 5 & 32 & 33 \\
32 & 13 & 28 & 10 & 8 & 13 & 24 & 10 & 19 & 15
\end{array}
$$

We need to find a linear combination $\lambda_{1} C_{f_{1}}^{(1)}+\lambda_{2} C_{f_{2}}^{(1)}+\lambda_{3} C_{f_{3}}^{(1)}$ of these first order partial derivatives matrices $C_{f_{1}}^{(1)}, C_{f_{2}}^{(1)}$ and $C_{f_{3}}^{(1)}$ that has rank 2. Thus we need to find $\lambda_{1}, \lambda_{2}, \lambda_{3}$ such that

$$
\begin{array}{r}
\lambda_{1}\left(\begin{array}{llllll}
32 & 17 & 30 & 25 & 11 & 24 \\
27 & 13 & 11 & 4 & 14 & 21 \\
15 & 11 & 11 & 7 & 5 & 34
\end{array}\right) \\
+\lambda_{2}\left(\begin{array}{rrrrrrr}
26 & 33 & 4 & 18 & 4 & 1 \\
35 & 36 & 4 & 13 & 10 & 32 \\
2 & 4 & 2 & 5 & 27 & 25
\end{array}\right) \\
+\lambda_{3}\left(\begin{array}{rrrrrr}
22 & 26 & 19 & 10 & 8 & 13 \\
13 & 20 & 8 & 35 & 20 & 19 \\
28 & 8 & 26 & 10 & 1 & 8
\end{array}\right)
\end{array}
$$


has rank 2 . We can identify a matrix of rank 2 by considering its 3 -minors, and, as an example, the first 3-minor of the above matrix is given by

$34 \lambda_{1}^{3}+22 \lambda_{1}^{2} \lambda_{2}+30 \lambda_{1}^{2} \lambda_{3}+19 \lambda_{1} \lambda_{2}^{2}+9 \lambda_{1} \lambda_{2} \lambda_{3}+9 \lambda_{1} \lambda_{3}^{2}+22 \lambda_{2}^{3}+20 \lambda_{2}^{2} \lambda_{3}+27 \lambda_{2} \lambda_{3}^{2}+5 \lambda_{3}^{3}$.

There are 153 -minors of the above $3 \times 6$ matrix, and we require them all to vanish for this matrix to have rank 2. This gives a system of 15 homogeneous cubic equations in $\lambda_{1}, \lambda_{2}, \lambda_{3}$. However, there are only 10 cubic monomials in 3 variables, so we can solve this system by direct linearisation to obtain $\lambda_{1}=\lambda_{3}$ and $\lambda_{2}=$ $26 \lambda_{3}$ as the unique solution. We thus consider the polynomial $g=f_{1}+26 f_{2}+f_{3}$ which has a vector of coefficients given by $(9,25,21,22,12,26,27,36,21,21)$ (with respect to lexicographic ordering) and partial derivatives matrix of rank 2

$$
C_{g}^{(1)}=C_{f_{1}}^{(1)}+26 C_{f_{2}}^{(1)}+C_{f_{3}}^{(1)}=\left(\begin{array}{rrrrrr}
27 & 13 & 5 & 22 & 12 & 26 \\
25 & 7 & 12 & 7 & 35 & 21 \\
21 & 12 & 15 & 36 & 5 & 26
\end{array}\right) .
$$

We can now either factor $g$ directly or by noting that any factor of $g$ is a linear combination (possibly over an extension field) of $27 x_{0}+25 x_{1}+21 x_{2}$ and $13 x_{0}+$ $7 x_{1}+12 x_{2}$, which are given by a basis for the column space of $C_{g}^{(1)}$. Thus we obtain a factorisation over $\mathrm{GF}(37)$ of a linear combination of $f_{1}, f_{2}, f_{3}$ as

$f_{1}+26 f_{2}+f_{3}=9\left(x_{0}+32 x_{1}+3 x_{2}\right)\left(x_{0}^{2}+16 x_{0} x_{1}+24 x_{0} x_{2}+29 x_{1}^{2}+24 x_{1} x_{2}+9 x_{2}^{2}\right)$

For a solution in $\operatorname{GF}(37)$, we obtain $x_{0}=-\left(32 x_{1}+3 x_{2}\right)$, which on substitution into $f_{1}$ gives (over $\mathrm{GF}(37)$ )

$$
0=x_{1}^{3}+x_{1}^{2} x_{2}+9 x_{1} x_{2}^{2}+33 x_{2}^{3}=\left(x_{1}+24 x_{2}\right)\left(x_{1}^{2}+14 x_{1} x_{2}+6 x_{2}^{2}\right) .
$$

Thus over GF(37) we obtain $x_{1}=-24 x_{2}=13 x_{2}$, so $x_{0}=-(32.13+3) x_{2}=25 x_{2}$. This gives $x_{1}=2 x_{0}$ and $x_{2}=3 x_{0}$, so the solution to the homogeneous cubic system $f_{1}=f_{2}=f_{3}=0$ is given by $\left(x_{1}, x_{2}, x_{3}\right)=\mu(1,2,3)$ for $\mu \in \mathrm{GF}(37)$. For comparison, calculating this solution using a Gröbner basis or an XL algorithm requires the generation of polynomials of degree 6 .

\section{B The $\mathcal{L} \mathcal{S}$-Criterion}

We let $\mathbb{F}=\operatorname{GF}(2)(\theta)$, where $\theta^{4}+\theta+1=0$, be a field with $2^{4}$ elements, and we represent elements of this field in hexadecimal, so, for example, C denotes $\theta^{3}+\theta^{2}$. We consider the solution of the homogeneous cubic system $f_{1}=f_{2}=f_{3}=0$ over $\mathbb{F}$ by applying the $\mathcal{L} \mathcal{S}$-criterion (Definition $\left[\right.$ ), where $f_{1}, f_{2}$ and $f_{3}$ are given below.

$$
\begin{aligned}
& 6 x_{0}^{3}+7 x_{0}^{2} x_{1}+9 x_{0}^{2} x_{2}+2 x_{0} x_{1}^{2}+\mathrm{C} x_{0} x_{1} x_{2}+5 x_{0} x_{2}^{2}+\mathrm{F} x_{1}^{3}+0 x_{1}^{2} x_{2}+\mathrm{D} x_{1} x_{2}^{2}+6 x_{2}^{3} \\
& \mathrm{~B} x_{0}^{3}+\mathrm{B} x_{0}^{2} x_{1}+\mathrm{D} x_{0}^{2} x_{2}+0 x_{0} x_{1}^{2}+8 x_{0} x_{1} x_{2}+4 x_{0} x_{2}^{2}+5 x_{1}^{3}+6 x_{1}^{2} x_{2}+3 x_{1} x_{2}^{2}+\mathrm{A} x_{2}^{3} \\
& \mathrm{E} x_{0}^{3}+9 x_{0}^{2} x_{1}+\mathrm{D} x_{0}^{2} x_{2}+\mathrm{D} x_{0} x_{1}^{2}+1 x_{0} x_{1} x_{2}+\mathrm{A} x_{0} x_{2}^{2}+1 x_{1}^{3}+3 x_{1}^{2} x_{2}+\mathrm{C} x_{1} x_{2}^{2}+5 x_{2}^{3}
\end{aligned}
$$

We wish to to find a linear combination $g=\lambda_{1} f_{1}+\lambda_{2} f_{2}+\lambda_{3} f_{3} \in \mathcal{R}_{\mathbb{F}, n}^{3}$. The $\mathcal{L} \mathcal{S}$-criterion for a cubic system is the $\mathcal{L}^{1} \mathcal{S}$ condition (Lemma 7 Part 1), that is 
$g \in U_{1}^{\prime}<W_{3}$. However, a polynomial in three variables in $W_{3}$ is in $U_{1}^{\prime}$ if and only if the coefficient of $x_{0} x_{1} x_{2}$ is zero, so we need to find $\lambda_{1}, \lambda_{2}, \lambda_{3}$ such that $\mathrm{C} \lambda_{1}+8 \lambda_{2}+1 \lambda_{3}=0$. Thus we have $\left(\lambda_{1}, \lambda_{2}, \lambda_{3}\right)^{T} \in\left\langle(1,0, \mathrm{C})^{T},(0,1,8)^{T}\right\rangle$. We can now define $f_{1}^{\prime}=f_{1}+\mathrm{C} f_{3}$ and $f_{2}^{\prime}=f_{2}+8 f_{3}$, and find a linear combination of $f_{1}^{\prime}$ and $f_{2}^{\prime}$ which factors either by direct search or by some other technique. We thus obtain

$f_{1}^{\prime}+9 f_{2}^{\prime}=f_{1}+9 f_{2}+8 f_{3}=3\left(x_{0}+x_{1}+2 x_{2}\right)\left(x_{0}^{2}+4 x_{0} x_{1}+9 x_{0} x_{2}+4 x_{1}^{2}+\mathrm{A} x_{1} x_{2}+\mathrm{B} x_{2}^{2}\right)$.

This gives $x_{0}=x_{1}+2 x_{2}$ as the unique solution over $\mathbb{F}$, and upon substitution into $f_{1}, f_{2}$ and $f_{3}$, we obtain the following two linearly independent equations:

$$
\begin{aligned}
& \mathrm{C} x_{1}^{3}+6 x_{1}^{2} x_{2}+\mathrm{E} x_{1} x_{2}^{2}+7 x_{2}^{3}=\mathrm{C}\left(x_{1}+7 x_{2}\right)^{2}\left(x_{1}+9 x_{2}\right) \\
& 5 x_{1}^{3}+5 x_{1}^{2} x_{2}+1 x_{1} x_{2}^{2}+0 x_{2}^{3}=5 x_{1}\left(x_{1}+5 x_{2}\right)\left(x_{1}+9 x_{2}\right) .
\end{aligned}
$$

We thus deduce that $x_{1}=9 x_{2}$ and $x_{0}=(9+2) x_{2}=\mathrm{B} x_{2}$, so the solution to $f_{1}=f_{2}=f_{3}=0$ is given by $\left(x_{0}, x_{1}, x_{2}\right)=\mu(4,2,1)$ for $\mu \in \mathbb{F}$. For comparison, calculating this solution using a Gröbner basis or an XL algorithm requires the generation of polynomials of degree 6 .

\section{A Quadratic System in Even Characteristic}

We use the field $\mathbb{F} \cong \mathrm{GF}\left(2^{4}\right)$ of Appendix $\mathrm{B}$. We consider the solution of the homogeneous quadratic system $f_{1}=\ldots=f_{7}=0$ using the method of Section 4.1. where $f_{1}, \ldots, f_{7} \in \mathbb{F}\left[x_{0}, \ldots, x_{6}\right]$. The coefficients of $f_{1}, \ldots, f_{7}$ are given with respect to the lexicographic ordering $x_{0}^{2}, x_{0} x_{1}, \ldots, x_{6}^{2}$ by the array below.

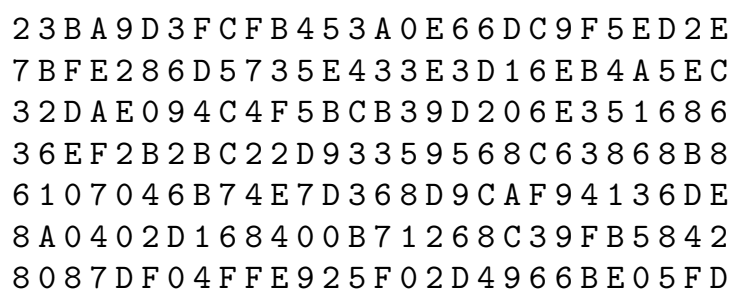

We wish to find $\lambda_{1}, \ldots, \lambda_{7}$ such that $g=\lambda_{1} f_{1}+\ldots \lambda_{7} f_{7} \in \mathcal{R}_{\mathbb{F}, n}^{2}$, so $g$ is given by

$$
\begin{aligned}
g= & \left(2 \lambda_{1}+7 \lambda_{2}+3 \lambda_{3}+3 \lambda_{4}+6 \lambda_{5}+8 \lambda_{6}+8 \lambda_{7}\right) x_{0}^{2} \\
& +\left(3 \lambda_{1}+\mathrm{B} \lambda_{2}+2 \lambda_{3}+6 \lambda_{4}+1 \lambda_{5}+\mathrm{A} \lambda_{6}+0 \lambda_{7}\right) x_{0} x_{1} \\
& +\left(\mathrm{B} \lambda_{1}+\mathrm{F} \lambda_{2}+\mathrm{D} \lambda_{3}+\mathrm{E} \lambda_{4}+0 \lambda_{5}+0 \lambda_{6}+8 \lambda_{7}\right) x_{0} x_{2}+\ldots \\
= & \Delta_{01} x_{0} x_{1}+\Delta_{02} x_{0} x_{2}+\ldots
\end{aligned}
$$

We can now use the 2-minor identity (Section 4.1)

$$
\mathcal{A}_{0,1,2,3}=\Delta_{01} \Delta_{23}+\Delta_{02} \Delta_{13}+\Delta_{03} \Delta_{12}=0
$$

to obtain a quadratic expression $Q\left(\lambda_{1}, \ldots, \lambda_{7}\right)=0$, where the coefficients of $Q$ are given with respect to the lexicographic ordering $\lambda_{1}^{2}, \lambda_{1} \lambda_{2}, \ldots, \lambda_{7}^{2}$ by the array

F 945 B A E 6371 D 954722 D 04 A 00488 A. 
There are $\left(\begin{array}{l}7 \\ 4\end{array}\right)=35$ such 2-minor identities giving rise to 35 quadratic expressions in $\lambda_{1}, \ldots, \lambda_{7}$ in total. As there are only 28 quadratic monomials in 7 variables, we can express this quadratic system as a linear system in 28 variables using a $35 \times 28$ matrix. This matrix has rank 27 and reducing it to echelon form gives the unique (up to multiplication) solution

$$
\left(\lambda_{1}, \lambda_{2}, \lambda_{3}, \lambda_{4}, \lambda_{5}, \lambda_{6}, \lambda_{7}\right)=(2, \mathrm{C}, 7,2, \mathrm{~F}, 2,1) .
$$

We can therefore obtain a linear combination of $f_{1}, \ldots, f_{7}$ which is a rank-2 product polynomial and so which factors to give

$$
\begin{aligned}
0 & =2 f_{1}+\mathrm{C} f_{2}+7 f_{3}+2 f_{4}+\mathrm{F} f_{5}+2 f_{6}+f_{7} \\
& =6\left(x_{0}+\mathrm{E} x_{1}+6 x_{2}+6 x_{3}+\mathrm{B} x_{4}+\mathrm{F} x_{5}+6 x_{6}\right)\left(x_{0}+9 x_{1}+\mathrm{D} x_{2}+\mathrm{C} x_{3}+2 x_{6}\right) .
\end{aligned}
$$

Thus we know that either $x_{0}+\mathrm{E} x_{1}+6 x_{2}+6 x_{3}+\mathrm{B} x_{4}+\mathrm{F} x_{5}+6 x_{6}=0$ or $x_{0}+9 x_{1}+\mathrm{D} x_{2}+\mathrm{C} x_{3}+2 x_{6}=0$. We can make these two substitutions to reduce the original problem in seven variables to one of two problems in six variables and so on. We thus find that the unique (projective) solution is $(1,2,4,8,3,6$, C). For comparison, calculating this solution using a Gröbner basis or an XL algorithm requires the generation of polynomials of degree 7 .

\section{A Quartic System in Even Characteristic}

We use the field $\mathbb{F} \cong \mathrm{GF}\left(2^{4}\right)$ of Appendix $\mathrm{B}$, We consider the solution of the homogeneous quartic system $f_{1}=f_{2}=f_{3}=f_{4}=f_{5}=0$ using the method of Section [4.2, where $f_{1}, \ldots, f_{5} \in \mathbb{F}\left[x_{0}, x_{1}, x_{2}, x_{3}, x_{4}\right]$ satisfy the $\mathcal{L} \mathcal{S}$-Criterion (without loss of generality). The coefficients of $f_{1}, \ldots, f_{5}$ are given below with respect to the lexicographic ordering $x_{0}^{4}, x_{0}^{3} x_{1}, \ldots, x_{4}^{4}$.

$$
\begin{aligned}
& \text { 49D32B5BD3D4AD69932C00A0A47A008C971A54E967A6950BB086D2FF0D95D8583B6103 } \\
& \text { 2D68B5ECOF085974058900407984F0A59C80E7924EBA6B03A069D8B9E4DC2A2E7634EB } \\
& \text { 6E03B1E544DF3352E19D00C082A850CFED40169539B0259520FD730402F6C18FCBDC6D } \\
& \text { 475365A26C3E7F0CC9EC00E030E8301361A220DCAA9EC573D00D369E441F2A9701149E } \\
& \text { D943065F4D8DB2F9629E00303CB9E0EDA6DF00E28151E25960567B58E1AB1441A76B8C }
\end{aligned}
$$

A rank-2 product polynomial in even degree is an element of the $\mathcal{L}^{2} \mathcal{S}$-subspace (Definition 3), and we note without loss of generality that $f_{1}, \ldots, f_{5} \in U_{1}$, the $\mathcal{L}^{2} \mathcal{S}$-subspace of $W_{4}$.

A linear combination $g=\lambda_{1} f_{1}+\lambda_{2} f_{2}+\lambda_{3} f_{3}+\lambda_{4} f_{4}+\lambda_{5} f_{5}$ is given by

$$
\begin{aligned}
g= & \left(4 \lambda_{1}+2 \lambda_{2}+6 \lambda_{3}+4 \lambda_{4}+\mathrm{D} \lambda_{5}\right) x_{0}^{4} \\
& +\left(9 \lambda_{1}+\mathrm{D} \lambda_{2}+\mathrm{E} \lambda_{3}+7 \lambda_{4}+9 \lambda_{5}\right) x_{0}^{3} x_{1} \\
& +\left(\mathrm{D} \lambda_{1}+6 \lambda_{2}+0 \lambda_{3}+5 \lambda_{4}+4 \lambda_{5}\right) x_{0}^{3} x_{2}+\ldots \\
= & \Gamma_{01} x_{0}^{3} x_{1}+\Gamma_{02} x_{0}^{3} x_{2}+\ldots
\end{aligned}
$$

We can now use the cubic identities $\mathcal{B}$ for $g$ to be a rank-2 product polynomial to obtain $\left(\begin{array}{l}5 \\ 3\end{array}\right) \times\left(\begin{array}{l}5 \\ 3\end{array}\right)=100$ homogeneous cubic equations in $\lambda_{1}, \ldots, \lambda_{5}$ (Section 4.2). For example, the coefficients with respect to the lexicographic ordering $\lambda_{1}^{3}, \lambda_{1}^{2} \lambda_{2}, \ldots, \lambda_{5}^{3}$ of the cubic expression given by $\mathcal{B}_{0,1,2,0,1,2}$ is given below. 
Thus we obtain 100 cubic expressions in the 35 cubic monomials in the variables $\lambda_{1}, \ldots, \lambda_{5}$, so we can express this cubic system as using a $100 \times 35$ matrix. This matrix has rank 34 and reducing it to echelon form gives the unique (projective) solution

$$
\left(\lambda_{1}, \lambda_{2}, \lambda_{3}, \lambda_{4}, \lambda_{5}\right)=(5,2,9, \mathrm{~B}, 1) .
$$

Thus we construct the linear polynomial $g=5 f_{1}+2 f_{2}+9 f_{3}+\mathrm{B} f_{4}+1 f_{5}$, with coefficents given below, which is a rank-2 product polynomial.

\section{DABAA51A9A5379A68200304EB690F755CD63ED76419BCD705A2B95FBB4017D5E6587}

We can indeed factor this linear combination of $f_{1}, \ldots, f_{5}$ to obtain

$$
0=g=\left(x_{0}+\mathrm{D} x_{1}+9 x_{2}+5 x_{3}+\mathrm{B} x_{4}\right)^{2} \times \text { Irreducible Quadratic. }
$$

A solution over $\mathbb{F}$ therefore satisfies $x_{0}+\mathrm{D} x_{1}+9 x_{2}+5 x_{3}+\mathrm{B} x_{4}=0$. We can thus make a substitution to reduce the original problem in five variables to a problem in four variables. We can continue using this techniques on the smaller system to give $(1, E, 4,6,7)$ as the unique (projective) solution to the original system. For comparison, calculating this solution using a Gröbner basis or an XL algorithm requires the generation of polynomials of degree 15 .

\section{E A Quintic System in Even Characteristic}

We use the field $\mathbb{F} \cong \mathrm{GF}\left(2^{4}\right)$ of Appendix $\mathrm{B}$. We consider the solution of the homogeneous quintic system $f_{1}=f_{2}=f_{3}=f_{4}=f_{5}=0$ using the method of Section [4.2. where $f_{1}, \ldots, f_{5} \in \mathbb{F}\left[x_{0}, x_{1}, x_{2}, x_{3}, x_{4}\right]$ satisfy the $\mathcal{L} \mathcal{S}$-Criterion (without loss of generality). The coefficients of $f_{1}, \ldots, f_{5}$ are given below with respect to the lexicographic ordering $x_{0}^{5}, x_{0}^{4} x_{1}, \ldots, x_{4}^{5}$.

$$
\begin{aligned}
& \text { 63DAD00009001040B6DB008048BE3078F 167000C007030000000000200C0300 } \\
& \text { 0070103023E90040612D405CC4ED007070000E0B074B30010421B010BD32D7A }
\end{aligned}
$$

OA2B2D000A0060B25D010010178D50FE5700000A00C030000000000F0010D00 006070909CAF0040552CD0EE87A900C030000C060EOF7407869930F0C078736

4BC09900030050E4933F00A0ECE25004F66700010080D0000000000B00B0300 0070A0E1FDC100E07D8A2050625300D0D000090C0251130D947EF030FCF0B16

8A0D52000A00505FD51800B0324DC0200F39000B009090000000000700B0B00 0010F0AB1A4C0070DC90D0B8CBFA00907000040C0E00DD07C0E790F03EFEE84

2416BD00050060FCC4FA00D078973004AFF50004003040000000000200D0900 0010D09EAB24008004FB104FBA2E008050000C070664130C76D58080812CDDF

A rank-2 product polynomial in odd degree is an element of the $\mathcal{L}^{1} \mathcal{S}$-subspace (Definition 4), and we note without loss of generality that $f_{1}, \ldots, f_{5} \in U_{2}^{\prime}$, the $\mathcal{L}^{1} \mathcal{S}$-subspace of $W_{5}$. 
A linear combination $g=\lambda_{1} f_{1}+\lambda_{2} f_{2}+\lambda_{3} f_{3}+\lambda_{4} f_{4}+\lambda_{5} f_{5}$ is given by

$$
\begin{aligned}
g= & \left(6 \lambda_{1}+0 \lambda_{2}+4 \lambda_{3}+8 \lambda_{4}+2 \lambda_{5}\right) x_{0}^{5} \\
& +\left(3 \lambda_{1}+\mathrm{A} \lambda_{2}+\mathrm{B} \lambda_{3}+\mathrm{A} \lambda_{4}+4 \lambda_{5}\right) x_{0}^{4} x_{1} \\
& +\left(\mathrm{D} \lambda_{1}+2 \lambda_{2}+\mathrm{C} \lambda_{3}+0 \lambda_{4}+1 \lambda_{5}\right) x_{0}^{4} x_{2}+\ldots \\
= & \Gamma_{00} x_{0}^{5}+\Gamma_{01} x_{0}^{4} x_{1}+\Gamma_{02} x_{0}^{4} x_{2}+\ldots
\end{aligned}
$$

We can now use the cubic identities $\mathcal{C}$ for $g$ to be a rank-2 product polynomial to obtain $\left(\begin{array}{l}5 \\ 3\end{array}\right) \times\left(\begin{array}{l}5 \\ 3\end{array}\right)=100$ homogeneous cubic equations in $\lambda_{1}, \ldots, \lambda_{5}$ (Section 4.2). For example, the coefficients with respect to the lexicographic ordering $\lambda_{1}^{3}, \lambda_{1}^{2} \lambda_{2}, \ldots, \lambda_{5}^{3}$ of the cubic expression given by $\mathcal{C}_{0,1,2,0,1,2}$ is given below.

\section{F34B6C6BAC6AF0B48FA8D2CD5BCADA}

Thus we obtain 100 cubic expressions in the 35 cubic monomials in the variables $\lambda_{1}, \ldots, \lambda_{5}$, so we can express this cubic system as using a $100 \times 35$ matrix. This matrix has rank 30 and reducing it to echelon form gives the following relations (amongst others):

$$
0=\lambda_{1}^{2} \lambda_{5}+\mathrm{A} \lambda_{5}^{3}=\lambda_{2}^{2} \lambda_{5}+1 \lambda_{5}^{3}=\lambda_{3}^{2} \lambda_{5}+\mathrm{E} \lambda_{5}^{3}=\lambda_{4}^{2} \lambda_{5}+7 \lambda_{5}^{3} .
$$

Thus analysing this equation system quickly shows that the unique (projective) solution is given by

$$
\left(\lambda_{1}, \lambda_{2}, \lambda_{3}, \lambda_{4}, \lambda_{5}\right)=(\mathrm{F}, 1, \mathrm{D}, 6,1) .
$$

Thus we construct the linear polynomial $g=\mathrm{F} f_{1}+1 f_{2}+\mathrm{D} f_{3}+6 f_{4}+1 f_{5}$, with coefficents given below, which is a rank-2 product polynomial.

\section{C3000C00E0ABD4AA0090F13E608A2B980004005080000000000700D0D00} OOF0A01CF25600EOCE1B40FCD25D00202000010F08351B0BE8E8C080A74B9D6

This linear combination of $f_{1}, \ldots, f_{5}$ factors to give

$$
0=g=\left(x_{0}+8 x_{1}+\mathrm{A} x_{2}+\mathrm{D} x_{3}+6 x_{4}\right) \times(\text { Irreducible Quadratic })^{2} .
$$

A solution over $\mathbb{F}$ therefore satisfies $x_{0}+8 x_{1}+\mathrm{A} x_{2}+\mathrm{D} x_{3}+6 x_{4}=0$. We can thus make a substitution to reduce the original problem in five variables to a problem in four variables. We can continue using this techniques on the smaller system to give $(1,4,4, \mathrm{~A}, \mathrm{E})$ as the unique (projective) solution to the original system. For comparison, calculating this solution using a Gröbner basis or an XL algorithm requires the generation of polynomials of degree 20 . 